\title{
Determining Flood Protection Strategy with Uncertain Parameter Using Adjustable Robust Counterpart Methodology
}

\author{
Diah Chaerani*), Muttaqien Rodhiya Robbi, Elis Hertini, Endang Rusyaman, Erick Paulus \\ Department of Mathematics FMIPA Universitas Padjadjaran \\ *E-mail: d.chaerani@unpad.ac.id
}

\begin{abstract}
Flooding is a natural disaster that often occurs, it is not surprising that floods are one of the problems that must be resolved in various countries, one of which is Indonesia. Flood is very detrimental to the public because the impact could be the loss of material and non-material. A flood protection system is needed and must be managed properly. This aims in management of flood protection systems often requires efficient cost control strategies that are the lowest possible long-term costs, but still meets the flood protection standards imposed by regulators in all plans. In this paper a flood protection strategy is modeled using Adjustable Robust Optimization. In this approach, there are two kinds of variables that must be decided, i.e., adjustable and non-adjustable variables. A numerical simulation is presented using Scilab Software.
\end{abstract}

Keywords: Flood Protection Strategy, Uncertainty, Adjustable Robust Optimization, Scilab Software

\section{INTRODUCTION}

Natural disasters have often been heard by everyone. According to Law No. 24 of 2007 on Disaster Management, natural disasters are defined as disasters caused by events or a series of events caused by nature including earthquakes, tsunamis, volcanic eruptions, floods, droughts, hurricanes, and landslides. Natural disasters such as volcanic eruptions, earthquakes, tsunamis, hurricanes, forest fires, landslides, floods and other natural disasters often occur in many countries around the world. Natural disasters that almost all countries have experienced and become the world's attention are flooding.

Flood sare an event where water in a channel increases and exceeds its capacity (Adi, 2013). Floods occur when the volume of water in a body of water, such as rivers, deltas, seas, overflows and causes water to come out of the boundary of the water body so that it soaks the surrounding land. Floods are caused by various trigger factors both from natural and human factors. Factors that causing floods are among others due to very high rainfall, the eradication of river water due to garbage and mud, wrong management of urban spatial planning and so forth. Floods have a bad impact on humans and even harm humans. These impacts can be either material or nonmaterial losses.

Indonesia is one country frequently hit by natural disasters, especially floods. Indonesian territory is located on the equator so that it receives a lot of sun heat and high rainfall, therefore Indonesia is prone to hydro meteorological natural disasters such as floods, droughts, large sea waves (Rianti, et al., 2016). Badan Nasional Penanggulangan Bencana (BNPB) noted that from the total hydrometeorological disasters that most often occur in Indonesia are floods and landslides (DIBI BNPB, 2012). The flood protection system is very important in flood management. The area at risk of flooding are protected by flood protection system, namely by ring dikes consisting of dikes, dams, dunes, plateaus, and other water defense building. This flood protection system must be managed properly, so that flood protection standards can be met and can prevent the occurrence of floods in the area. According to Postek, et al., (2016), the management of flood protection systems often requires efficient cost control strategies that are the lowest possible long-term costs, but still meet the flood protection standards imposed by regulators in all plans.

According to Postek, et al. (2016), there are two challenges must be solved in modeling in this paper. First, uncertainty. Many of the important parameters that the underlying strategy (eg sea level rise) is uncertain, and can only be known in the future. This creates a challenge called robustness in the uncertainty of parameters. Therefore, according to Ben-Tal \& Nemirovski (2002), optimization problems involving this uncertainty can be overcome by using robust optimization because it guarantees the problem is computationally tractability. Decisions applied at the next stage need to be 
adjusted to the uncertainty values revealed. In other words, every decision that will be applied depends on the previous stages. So this problem is categorized as a multistage optimization problem. This creates a challenge called adjustability to the uncertainty revealed. Therefore, according to Gorisson et al. (2015), multistage optimization problems can be overcome by using Adjustable Robust Counterpart (ARC).

The subject of this paper is to analyze how the adjustable robust optimization model addresses the problem of flood protection strategies with uncertainty parameters.In this paper, we discuss how the optimization model with uncertainty and adjustability. Then a numerical simulation was carried out with data obtained from the journal Eijgenraam, et al. (2012). Scilab software is used to assist in conducting numerical simulations in this paper.

\section{METHODS}

\section{Robust Optimization}

According to Gorissen, et al. (2015), Robust Optimization (RO) does not assume that the probability distribution is known, but instead assumes that uncertainty data is in what is called the uncertainty set.According to Gorissen, et al. (2015), the general forms of robust optimization model are as follows:

$$
\begin{aligned}
& \min _{x} c^{\mathrm{T}} x \\
& \text { s.t. } A x \leq b \\
& (c, A, b) \in U
\end{aligned}
$$

where $c \in \mathfrak{R}^{n}, \quad A \in \mathfrak{R}^{m \times n}$, and $b \in \mathfrak{R}^{m}$ are the uncertainty coefficients, and $U$ isthe uncertainty set.

The robust optimization approach to changing uncertainty problems (2.1) becomes a single deterministic problem called the Robust Counterpart (RC), namely:

$$
\pi^{*}=\left\{\min _{x} c^{\mathrm{T}} x: A x \leq b ;(c, A, b) \in U\right\}
$$

A vector $x^{*}$ is called a robust solution if for all realization $(c, A, b) \in U, \quad x^{*}$ is feasible, and the objective function value is guaranteed by $\pi^{*}$ the largest value.

The constraint in equation (2.2) can be changed by considering the semi-definite robustness of canonics as follows.

$a^{T} x-b \leq 0, \quad \forall(a, b) \in U$

where $a$ is a vector in $\Re^{n}$ dan $b$ is a scalar representing $a_{i}$ and $b_{i}$. While $U$ is representing $U_{i}$. Uncertainty parameter $a$ and $b$, as well as set uncertainty $U$ can be converted into a primitive factor form $\zeta \in \mathfrak{R}^{L}$, so that it can be written as $a=\bar{a}+P \zeta, b=\bar{b}+p^{\mathrm{T}} \zeta$,

$U=\left\{\left(\begin{array}{c}a=\bar{a}+P \zeta \\ b=\bar{b}+p^{\mathrm{T}} \zeta\end{array}\right) \mid \zeta \in Z\right\}$

where vector $\bar{a} \in \mathfrak{R}^{n}$ and scalar $\bar{b} \in \mathfrak{R}$ is called nominal, matrix $P \in \mathfrak{R}^{n \times L}$ and vector $p \in \mathfrak{R}^{L}$, and set $Z \subset \mathfrak{R}^{L}$ is an uncertainty set for primitive factor. Then equation (2.4) can be substituted in equation (2.3) by substituting $a$ and $b$ in expectation $\zeta$ to be

$\left(\bar{a}^{\mathrm{T}} x-\bar{b}\right)+\left(P^{\mathrm{T}} x-p^{\mathrm{T}}\right)^{T} \zeta \leq 0, \quad \forall \zeta \in Z$

representation of uncertainty by this primitive factor is not required, but only for ease. To generate a $U$ which meets the representation (3.6), the uncertainties in $a$ and $b$ are described by a simple interval, namely:

$U=\left\{(a, b) \mid a^{l} \leq a \leq a^{u}\right.$ dan $\left.b^{l} \leq b \leq b^{u}\right\}$

with defined

$\bar{a}=\frac{a^{l}+a^{u}}{2}, \quad P=\operatorname{diag}\left(\frac{a^{u}-a^{l}}{2}\right), \quad \bar{b}=\frac{b^{l}+b^{u}}{2}$,

$p=\frac{b^{u}-b^{l}}{2}$,

$Z=\left\{\zeta \mid-1 \leq \zeta_{l} \leq 1, l=1, \mathrm{~K}, L\right\}$.

According to den Hertog, et al. (2015), this Robust Counterpart cannot be solved directly with a standard optimization method. The challenge in Robust Optimization is to find the type of uncertainty set that can be formulated as a "tractable" optimization problem.

\section{Adjustable Robust Counterpart (ARC)}

According to Gorissen, et al. (2015), in the problem of multistage optimization, a basic understanding of robust optimization states that a "here and now" decision can be relaxed.Ben-Tal, et al. (2009) used parametric desicion rules for continuous adjustable variables. Whereas the technique cannot be applied to an adjustable integer variable. Adjustable Robust Counterpart (ARC) is given as follows:

$\min c(x, y, z)$

$x, y, z$

$$
\text { s.t. } A(\zeta) x+B(\zeta) y+C(\zeta) z<b ; \forall \zeta \in Z
$$

where $x \in \mathfrak{R}^{n_{1}}$ and $y \in \mathfrak{R}^{n_{2}}$ are the first stage decision "here and now" variable created before $\zeta \in \mathfrak{R}^{L}$ is realized, $z \in \mathfrak{R}^{n_{3}}$ shows the second stage decision "wait and see" variable which can be adjusted to the actual data, and $A(\zeta) \in \mathfrak{R}^{m_{1} \times n_{1}}$ and $B(\zeta) \in \mathfrak{R}^{m_{2} \times n_{2}}$ shows a matrix of the uncertainty coefficients "here and now" variable and $C(\zeta) \in \mathfrak{R}^{m_{3} \times n_{3}}$ shows the matrix of the uncertainty 
coefficient "wait and see" variable. To get the Adjustable Robust Counterpart Model with integer variables, previously the uncertainty set, $Z$,must be divided into $m$ subsets, $Z_{i}(i=1 \mathrm{~K}, m)$ where $Z=\bigcup_{i \in\{1, \mathrm{~K}, d\}} Z_{i}$. Then, variable $z_{i} \in \mathrm{Z}^{n_{3}}(i=1, \mathrm{~K}, m)$ is added where the decision is in the uncertainty subsets $Z_{i}$. Then, the objective function and the constraint function in equation (2.7) are replicated for each $Z_{i}$ anduncertainty subsets $Z_{i}$ and without reducing the generality of being

$\min _{x, y, z, t} t$

s.t. $c\left(x, y, z_{i}\right) \leq t ; \quad \forall i \in\{1, \mathrm{~K}, m\}$

$A(\zeta) x+B(\zeta) y+C(\zeta) z_{i} \leq b$

$$
\forall \zeta \in Z_{i}, \forall i \in\{1, \mathrm{~K}, m\}
$$

According to Gorissen, et al. (2015), Adjustable Robust Counterpart Optimization (ARC) produces more flexible decisions that can be adjusted according to the realization of the amount of data at a certain stage and produces the optimal robust objective value that is as good as the standard Robust Optimization approach. Dividingthe main uncertaintyset $Z$ intoseveral uncertainty subsets $Z_{i}$ can increase objective value by giving more freedom in making customized decisions, but the decision maker must make a balance between optimalization and computational complexity.

\section{Box Uncertainty Set and Ellipsoidal Uncertainty} Set

According to den Hertog, et al. (2015), first robust counterpart formulations are "tractable" for linear robust optimization problems withthe uncertainty box regions. One of the assumptions discussed earlier is uncertainty is constraint-wise, so focus on one constraint (and omit index $i$ ):

$(\bar{a}+P \zeta)^{\mathrm{T}} x \leq b, \quad \forall \zeta:\|\zeta\|_{\infty} \leq \rho$

$x$ satisfies equation (2.9) if and only if $x$ fulfills

$\bar{a}^{\mathrm{T}} x+\rho\left\|P^{\mathrm{T}} x\right\|_{1} \leq b$

Second, robust counterpart formulations are "tractable" for linear robust optimization problems with the uncertainty ellipsoidal regions. The form of the Robust Counterpart becomes

$(\bar{a}+P \zeta)^{\mathrm{T}} x \leq b, \quad \forall \zeta:\|\zeta\|_{2} \leq \rho$

$x$ satisfies equation (2.11) if and only if $x$ fulfills

$\bar{a}^{\mathrm{T}} x+\rho\left\|P^{\mathrm{T}} x\right\|_{2} \leq b$

\section{RESULTS AND DISCUSSION}

Adjustable Robust Optimization Model for Flood Protection Strategies

Deterministic model of the problem of flood protection strategies based can be seen in Postek, et al. (2016). In this section, the Adjustable Robust Optimization Model for Flood Protection Strategies is discussed.

$$
\begin{aligned}
& \min _{x, y} \sum_{t=1}^{T} \frac{1}{(1+d)^{t}}\left[\sum_{s=1}^{N_{s}} \sum_{h=1}^{N_{h}} p_{s, h} x_{t, s, h}+\sum_{m=1}^{N_{m}} p_{m} y_{t, m}\right] \\
& \text { s.t. } \sum_{\tau=1}^{t}\left[\sum_{h=1}^{N_{h}} h x_{t, s, h}+\sum_{m=1}^{N_{m}} a_{m, s, \tau, t} y_{t, m}\right] \geq n_{s, t}, \forall s, t \\
& \sum_{h=1}^{N_{h}} x_{t, s, h} \leq 1, \forall t, s \\
& x_{t, s, h}, y_{t, m} \in\{0,1\}, \forall t, s, h, m
\end{aligned}
$$

The relative dike height requirements are values that have been previously known. According to Postek, et al. (2016), actually not only based on flood protection standards, but also on sea level rise. So it can be defined as

$$
n_{s, t}=n_{s, 0}+\beta_{s} \sum_{\tau=1}^{t} r_{\tau}
$$

The first step carried out in the Adjustable Robust Optimization method is to determine which parameters are indeterminate parameters. In the matter of this flood protection strategy, according to Postek, et al. (2016), sea level rise may change to be different from the predicted value. Therefore, it can be assumed that the indeterminate parameter in the problem of this flood protection strategy is the parameter of sea level rise,. So taking into account this uncertainty factor, the parameter must be changed by assuming to be

$$
r_{\tau}=\bar{r}_{\tau}+\rho_{\tau} \zeta, \quad \forall \zeta \in Z \subseteq U
$$

Where the coefficient $\bar{r}_{\tau} \in \Re^{n}$ is a nominal data vector and is a confounding vector. The objective function on the problem of flood protection strategies is not affected by indeterminate parameters, so the assumption that the objective function is certainly fulfilled.

The second step carried out in the Adjustable Robust Optimization method is to determine the past variable which is an adjustable variable. In the matter of this flood protection strategy, according to Postek, et al. (2016), a large-scale action plan is carried out if something happens that causes flood protection standards not met. For example, a plan to repair or replace an embankment must be carried out if there is a small or large damage that requires repairs or replacement of 
the embankment. Therefore, this large-scale action plan carried out must adjust to the situation at a later stage of time. According to Gorissen, et al. (2015), this is called a "wait and see" decision and is not a "here and now" decision, so that the large-scale action plan decision variable, is an adjustable variable. Large-scale action plan decision variables,, are assumed to be in uncertainty. The uncertainty set is divided into subsets, wherein, the adjustable integer variable, is changed to where the decision is in the set of uncertainty parts.

$\min _{x, y, f} f$

$$
\begin{aligned}
& \text { s.t. } \sum_{t=1}^{T} \frac{1}{(1+d)^{t}}\left[\sum_{s=1}^{N_{s}} \sum_{h=1}^{N_{h}} p_{s, h} x_{t, s, h}+\sum_{m=1}^{N_{m}} p_{m} y_{t, m, i}\right]-f+u_{1}=0, \quad i=1, \ldots, d \\
& \sum_{\tau=1}^{t}\left[\sum_{h=1}^{N_{h}} h x_{t, s, h}+\sum_{m=1}^{N_{m}} a_{m, s, \tau, t} y_{t, m, i}\right]-v_{m}=n_{s, 0}+\beta_{s} \sum_{\tau=1}^{t}\left[\bar{r}_{\tau}+\rho_{\tau} \zeta\right], \quad \begin{array}{l}
\forall s, t, \\
i=1, \ldots, d
\end{array} \\
& \sum_{h=1}^{N_{h}} x_{t, s, h}+w_{n}=1 \\
& x_{t, s, h}, y_{t, m, i} \in\{0,1\} \text {, } \\
& v_{m}, w_{n} \geq 0 \text {, } \\
& f, u_{1} \geq 0 \text {, } \\
& \zeta \in Z_{i} \subseteq Z \subseteq U \text {, } \\
& \forall t, s \\
& \forall t, s, h \\
& \forall m, n
\end{aligned}
$$

where $v_{m}$ is the surplu svariable with $m \in N_{s} \times T$ and $w_{n}$ is theslackvariable with $n \in T \times N_{s}, f$ is the certain variableand $u_{1}$ is the slack variable.

The model above shows the problem of a flood protection strategy that involves uncertainty. The problem of flood protection strategies has an objective function, which is to minimize the total cost of all actions to be taken. Actions to be taken in accordance with flood protection standards. These actions include raising dikes, and other large-scale actions, such as repairing and replacing dikes, changing the distribution of river water flow, and others. The relative dike height requirement in the second obstacle is influenced by sea level rise whose value is uncertain.

$$
\begin{aligned}
& \min _{x, y, f} f \\
& \text { s.t. } \sum_{t=1}^{T} \frac{1}{(1+d)^{t}}\left[\sum_{s=1}^{N_{s}} \sum_{h=1}^{N_{h}} p_{s, h} x_{t, s, h}+\sum_{m=1}^{N_{m}} p_{m} y_{t, m, 1}\right]-f+u_{1}=0
\end{aligned}
$$

Adjustable Robust Counterpart (ARC) Formulation for Flood Protection Strategies Problem with box uncertaintyset.

Furthermore, it assumes that the uncertainty parameter in the Adjustable Robust Counterpart (ARC) formulation of the flood protection strategy is within the set of box uncertainty. Define the set of uncertainty boxes as follows:

$$
\begin{aligned}
Z=\left\{\zeta:\|\zeta\|_{\infty} \leq 1\right\} & \Leftrightarrow Z=\{\zeta:|\zeta| \leq 1\} \\
& \Leftrightarrow Z=\{\zeta:-1 \leq \zeta \leq 1\}
\end{aligned}
$$

Then suppose the set of uncertainty $Z$ is divided into a set of uncertainty parts $Z_{1}$ and $Z_{2}$, where

$Z_{1}=\{\zeta:-1 \leq \zeta \leq 0\}$ and $Z_{2}=\{\zeta: 0<\zeta \leq 1\}$ (3.6)

This implies that for $i=1$, the Robust Counterpart becomes the following problem. 


$$
\begin{array}{ll}
{\left[\sum_{\tau=1}^{t} \sum_{h=1}^{N_{h}} h x_{t, s, h}+\sum_{m=1}^{N_{m}} a_{m, s, \tau, t} y_{t, m, 1}\right]-v_{m}=n_{s, 0}+\beta_{s} \sum_{\tau=1}^{t} \bar{r}_{\tau},} & \forall s, t \\
\sum_{h=1}^{N_{h}} x_{t, s, h}+w_{n}=1, & \forall t, s \\
x_{t, s, h}, y_{t, m, 1} \in\{0,1\}, & \forall t, s, h \\
v_{m}, w_{n} \geq 0, & \forall m, n \\
f, u_{1} \geq 0 &
\end{array}
$$

For the case with $i=2$, the RC becomes

$\min _{x, y, f} f$

$$
\begin{array}{lll}
\text { s.t. } & \sum_{t=1}^{T} \frac{1}{(1+d)^{t}}\left[\sum_{s=1}^{N_{s}} \sum_{h=1}^{N_{h}} p_{s, h} x_{t, s, h}+\sum_{m=1}^{N_{m}} p_{m} y_{t, m, 2}\right]-f+u_{2}=0 & \\
& {\left[\sum_{\tau=1}^{t} \sum_{h=1}^{N_{h}} h x_{t, s, h}+\sum_{m=1}^{N_{m}} a_{m, s, \tau, t} y_{t, m, 2}\right]-v_{m}=n_{s, 0}+\beta_{s} \sum_{\tau=1}^{t}\left[-\bar{r}_{\tau}+\alpha \rho_{\tau}\right],} & \forall s, t \\
& \sum_{h=1}^{N_{h}} x_{t, s, h}+w_{n}=1, & \\
& x_{t, s, h}, y_{t, m, 2} \in\{0,1\}, & \forall t, s \\
& v_{m}, w_{n} \geq 0, & \forall t, s, h, m \\
& f, u_{2} \geq 0 & \forall m, n
\end{array}
$$

Furthermore,

Adjustable

Robust Protection Strategies Problem with ellipsoidal Counterpart (ARC) Formulation for Flood uncertainty set as a whole are as follows: For $i=1$,

$\min _{x, y, f} f$

$$
\begin{array}{ll}
\text { s.t. } \sum_{t=1}^{T} \frac{1}{(1+d)^{t}}\left[\sum_{s=1}^{N_{s}} \sum_{h=1}^{N_{h}} p_{s, h} x_{t, s, h}+\sum_{m=1}^{N_{m}} p_{m} y_{t, m, 1}\right]-f+u_{1}=0 & \\
{\left[\sum_{\tau=1}^{t} \sum_{h=1}^{N_{h}} h x_{t, s, h}+\sum_{m=1}^{N_{m}} a_{m, s, \tau, t} y_{t, m, 1}\right]-v_{m}=n_{s, 0}+\beta_{s} \sum_{\tau=1}^{t} \bar{r}_{\tau},} & \forall s, t \\
\sum_{h=1}^{N_{h}} x_{t, s, h}+w_{n}=1, & \\
x_{t, s, h}, y_{t, m, 1} \in\{0,1\}, & \forall t, s \\
v_{m}, w_{n} \geq 0, & \forall t, s, h, m
\end{array}
$$$$
f, u_{1} \geq 0
$$

For $i=2$,

$\min _{x, y, f} f$

$$
\text { s.t. } \sum_{t=1}^{T} \frac{1}{(1+d)^{t}}\left[\sum_{s=1}^{N_{s}} \sum_{h=1}^{N_{h}} p_{s, h} x_{t, s, h}+\sum_{m=1}^{N_{m}} p_{m} y_{t, m, 2}\right]-f+u_{1}=0
$$




$$
\begin{array}{ll}
{\left[\sum_{\tau=1}^{t} \sum_{h=1}^{N_{h}} h x_{t, s, h}+\sum_{m=1}^{N_{m}} a_{m, s, \tau, t} y_{t, m, 2}\right]-v_{m}=n_{s, 0}+\beta_{s} \sum_{\tau=1}^{t}\left[\bar{r}_{\tau}+\rho_{\tau}\right],} & \forall s, t \\
\sum_{h=1}^{N_{h}} x_{t, s, h}+w_{n}=1, & \forall t, s \\
x_{t, s, h}, y_{t, m, 2} \in\{0,1\}, & \forall t, s, h, m \\
v_{m}, w_{n} \geq 0, & \forall m, n \\
f, u_{1} \geq 0 & \\
\end{array}
$$

\section{Numerical Simulation}

The problem model of flood protection strategy that has been formulated in theform of Adjustable Robust Counterpart is applied using a case study. Suppose there are $N_{s}=3$ dike segments, ie dike 10 (first dike), dike 11 (second dike), and the dike 15 (third dike). In each dike segment, the number of possible dike increases is $N_{h}=3$ where the increase in the dike is $\{100,200,300\} \mathrm{cm}$.

The large-scale measures involved in this case study is the plan to repair the damaged dike. Assumed that $N_{m}=3$. It is also assumed that time is limited by $T=\{1,2\}$ years. The data used in this case study is sourced from the journal Eijgenraam, et al. (2012) with the title "Flood Prevention by Optimal Dike Heightening" which can be seen in Table 4.1.

Table 4.1. The Cost of Dike Heightening $h$ for Segment $S$

\begin{tabular}{cccc}
\hline \multirow{2}{*}{ Dike $(s)$} & \multicolumn{3}{c}{ The Cost of Dike Heightening $\left(p_{s, h}\right)$} \\
\cline { 2 - 4 } & $h=100 \mathrm{~cm}$ & $h=200 \mathrm{~cm}$ & $h=300 \mathrm{~cm}$ \\
\hline 1 & 91.18669 & 187.69112 & 311.14054 \\
2 & 213.3 & 383.98 & 554.66 \\
3 & 634.9991 & 2491.8794 & 8770.9412 \\
\hline
\end{tabular}

Then, the repair plan for the damaged dike is defined as $m=1$ shows the repair plan on the damaged dike 10 (first dike), $m=2$ shows the repair plan on the damaged dike 11 (second dike), and 3 shows the repair plan on the damaged dike 15 (third dike). Based on simulation data, it can be seen in Table 4.2, the costs are obtained for the plan to repair damaged dikes.

Table 4.2. The Cost of Damaged Dike Repair $m$

\begin{tabular}{cc}
\hline The large-scale measures $(m)$ & The Cost of Damaged Dike Repair $\left(p_{m}\right)$ \\
\hline 1 & 246.95614 \\
2 & 616.32898 \\
3 & 9271.3268 \\
\hline
\end{tabular}

It is assumed that the first year of the dike was not damaged so it is not necessary dike repairs and the impact of its application did not exist. The impacts of implementing a plan to repair damaged dikes in the second year can be seen in Table 4.3.

Table 4.3. Impact of Implementing the Dike Repair Plan $m$ in the Second Year on the Relative Dike Height at Dike $S$ in the Second Year

\begin{tabular}{cccc}
\hline \multirow{2}{*}{$\begin{array}{c}\text { Action Plans } \\
(m)\end{array}$} & \multicolumn{3}{c}{ Impact of Plans $\left(a_{m, s, \tau, t}\right)$} \\
\cline { 2 - 4 } & Dike -1 & Dike-2 & Dike-3 \\
2 & 200 & 0 & 0 \\
3 & 0 & 200 & 0 \\
& 0 & 0 & 200 \\
\hline
\end{tabular}


The model of flood protection strategy problem which has been reformulated in the form of Adjustable Robust Counterpart involves uncertainty parameters, namely parameters of sea level rise $r_{\tau}$ where in the previous sub-section $r_{\tau}$ has been defined. This parameter value is obtained by using the simple interval based on den Hertog, et al. (2015), so that with data from the journal Eijgenraam, et al. (2012) can be seen in Table 4.4.

The aim of the flood protection strategies is to create a schedule when to take various measures that minimize the present value of the measures costs such that at each time moment and at each dike segment, the flood protection standards are satisfied.(Postek, et al., 2016).
The numerical simulation result is that the cost required in this flood protection strategy is 2945.7217 million euros for the deterministic model, 3532.814 million euros for the worstcase in the robust optimization model with box uncertainty set (with $\alpha=10 \%$ ), and 3781,2366 million euros for the worst-case in the adjustable robust optimization model with ellipsoidal uncertainty set.

The optimal Schedule for dike heightening 10, 11 and 15 of the Deterministic Model, the Adjustable Robust Optimization Model with Uncertainty Box Set, the Adjustable Robust Optimization Model with Uncertainty Ellipsoidal Set can be seen in Table 4.1-4.3.

Table 4.4. The Parameter Value $\bar{r}_{\tau}$ and $\rho_{\tau}$

\begin{tabular}{ccccc}
\hline$\tau$ & $r_{\tau}^{l}$ & $r_{\tau}^{u}$ & $\bar{r}_{\tau}$ & $\rho_{\tau}$ \\
\hline 1 & 0.294 & 1.06 & 0.677 & 0.383 \\
2 & 0.588 & 2.12 & 1.354 & 0.766
\end{tabular}

Table 4.5. Schedule for Dike Heightening 10, 11 and 15 of the Deterministic Model

\begin{tabular}{cccc}
\hline \multirow{2}{*}{ Years } & \multicolumn{3}{c}{ Dike Heightening(cm) } \\
\cline { 2 - 4 } & Dike 10 & Dike 11 & Dike 15 \\
\hline 1 & 200 & 200 & 200 \\
2 & - & - & - \\
\hline
\end{tabular}

Table 4.6. Schedule of Dike Heightening 10, 11, and 15 of the Adjustable Robust Optimization Model with Uncertainty Box Set

\begin{tabular}{cccc}
\hline \multirow{2}{*}{ Years } & \multicolumn{3}{c}{ Dike Heightening $(\mathrm{cm})$} \\
\cline { 2 - 4 } & Dike 10 & Dike 11 & Dike 15 \\
\hline 1 & 200 & 200 & 200 \\
2 & - & - & 100 \\
\hline
\end{tabular}

Table 4.7. Schedule of Dike Heightening 10, 11, and 15 of the Adjustable Robust Optimization Model with Uncertainty Ellipsoidal Set

\begin{tabular}{cccc}
\hline \multirow{2}{*}{ Years } & \multicolumn{3}{c}{ Dike Heightening $(\mathrm{cm})$} \\
\cline { 2 - 4 } & Dike 10 & Dike 11 & Dike15 \\
\hline 1 & 200 & 300 & 200 \\
2 & - & - & 100 \\
\hline
\end{tabular}

Based on the simulation results above, the dike heightening schedule on dikes 10,11 and 15 with the deterministic model and the adjustable robust optimization model have some differences. The dike heightening costs required in the deterministic model also have lower costs compared to the dike heightening costs required in the adjustable robust optimization model. This is because the adjustable robust optimization model involves a factor in uncertain sea level rise. Sea level rise is causing increased flood protection standard, so it should be more measures must be taken to fulfill the flood protection 
standards. This causes the costs required to implement the measures will be even greater.

The flood protection strategy is made in order to the flood protection system can work well so that people can live more safely and comfortably. If viewed only in terms of costs, the adjustable robust optimization model is indeed greater than the deterministic model. But when viewed in terms of safety and security standards, the adjustable robust optimization model is much safer than the deterministic model. Beside the problem of flood protection strategies must in fact involve all factors that can affect flood protection standards. One of them is sea levels rise.Although only by involving the sea level rise factor, the adjustable robust optimization model can better describe the actual problem state. Therefore, it can be said that the adjustable robust optimization model is better compared to the deterministic model.

\section{CONCLUSION}

The Adjustable Robust Optimization model on the flood protection strategies problem involves the uncertainty factor of sea level rise which is an uncertainty parameter.This Adjustable Robust Optimization model is solved by approaching the box uncertainty set and the ellipsoidal uncertainty set. The Robust Adjustable Optimization Model produces a form of Linear Programming, so that the Adjustable Robust Optimization model is guaranteed to be computationally tractable. The numerical simulation results of the Adjustable Robust Optimization model on the flood protection strategies problem with sea level rise as an uncertainty parameter show the schedule of when every measures must be taken so that the cost of implementing the measures can be as minimal as possible,but still fulfill the flood protection standards.

\section{ACKNOWLEDGEMENT}

This research is funded by Penelitian Dasar Kemenristekdikti for the year of 2019 under Contract Number 01/E/KPT/2019 and 2923/UN6.D/LT/2019.

\section{REFERENCES}

Adi, S., 2013. Karakterisasi Bencana Banjir Bandang. Jurnal Sains dan Teknologi Indonesia, 15(1): 42-51.

Ben-Tal, A., Ghaoui, L. E. \& Nemirovski, A., 2009. Robust Optimization. s.1.:Princeton University Press.

A. Ben-Tal and A. Nemirovskii., 2002., Robust Optimization Methodology and Applications. Mathematical Programming, 92(3, Ser. B):453-480.

Eijgenraam, C., Brekelmans, R., den Hertog, D \& Roos, K., 2012. Flood Prevention by Optimal Dike Heightening. Working paper, 17.

Gorissen, B. L., Yanıkoglu, I. \& den Hertog, D, 2015. A Practical Guide to Robust Optimization. Omega, 53(2015):124-137.

den Hertog, D, A. Ben-Tal, A., Brekelmans., 2015, Practical Robust Optimization, Lecture Notes LNMB Course, Spring 2015.

Postek, K., den Hertog, D, Kind, J. \& Pustjens, C., 2016. Adjustable Robust Strategies For Flood Protection. (CentER Discussion Paper; Vol. 2016-038).

Rianti, R., Rahardjo, E. \& Zainuddin, M., 2016. Model Pengembangan Kesiapsiagaan Masyarakat Percontohan dan NonPercontohan Program Kesiapsiagaan Bencana Berbasis Masyarakat (KBBM) dalam Menghadapi Ancaman Banjir. Jurnal Dialog Penanggulangan Bencana, 7(2): 128-139.

DIBI BNPB (Data Informasi Bencana Indonesia), 2010. 